\title{
Research on Wushu Teaching Reform in Primary and Secondary Schools Under the Background of Modern Information Technology
}

\author{
Wang Jianli \\ college of physical education, Xianyang Normal University, Xianyang, Shaanxi, China. \\ Wang2003007@163.com

\begin{abstract}
With the development of the times, it is an inevitable trend to use modern information technology to change the traditional education mode. Using the method of literature, logical analysis and other scientific research methods, the paper analyzes the role and influence of modern information technology on Wushu Teaching in primary and secondary schools, and give some advice to the teachers for Wushu teaching. The conclusion: The application of modern information technology promotes the Wushu teachers' role change and give some advice to Wushu teacher for teaching methods modernized, and it plays an important role in promoting the cultivation of students' Wushu cognition and learning interest. Through the reform of Wushu Teaching under the condition of modern information technology, it enrich Wushu teaching methods, promote the improvement of Wushu teaching level, inherit and carry forward the national spirit and traditional sports culture.
\end{abstract}

Keywords: modern information technology, Chinese wushu, reform in teaching

\section{现代信息技术背景下中小学武术教学改革探析}

王建利

咸阳师范学院体育学院, 咸阳, 陕西, 中国

wang2003007@163. com

\section{摘要}

随着时代的发展，使用现代信息技术改变传统教育模式是必然的趋势。现代信息技术推动了新型教育模式的构 建, 为现代信息化课堂教学模式搭建了广阔的平台,通过信息化课堂教学改革, 向学习者全方位传递教育信息。 本文通过文献资料法、逻辑分析法等科研方法, 以现代信息技术与武术教学的特点相结合为契合点, 对现代信 息技术对中小学武术教学的作用与影响进行分析以及现代信息技术在武术教学方面的运用进行探析。认为: 现 代信息技术的应用, 促进了武术教师在教学中的角色与教学方法转变, 对学生的武术认知、学习兴趣的培养具 有重要的促进作用。通过现代信息技术条件下武术教学的改革, 丰富武术教学方法, 促进学校武术教学水平的 提高，传承和弘扬了民族精神和传统体育文化。

关键词: 现代信息技术; 武术; 教学改革

\section{1. 前言}

随着社会的发展，现代信息技术涉及到各个领域 中, 给全人类带来了巨大的影响, 改变了人们的工作、 学习、生活的方式。现代信息技术走进了校园, 构建
了新型的教学体制, 改变了教师的教学方式、学生的 学习方法, 现代信息技术使武术教学课堂生动活跃。 本文通过文献资料法、逻辑分析法等科研方法，以现 代信息技术与武术教学的特点相结合为契合点, 对现 代信息技术对中小学武术教学的作用与影响进行分 析以及现代信息技术在武术教学方面的运用进行探 
析。

\section{2. 现代信息技术在中小学武术教学中的应用 价值与意义}

现代信息技术以计算机、微电子和通讯技术为特 征的技术方法和手段来处理信息和进行信息管理。近 些年来, 在我国各级学校, 由于国家对于现代信息技 术进入教育领域的重视与积极推广, 投入了大量的人 力、物力资源, 教育信息技术已经得到了广泛的普及, 以 “互联网 + ”为模式的现代信息技术在教育教学 得到了重视和应用。

在学校体育教学方面, 现代信息技术也逐步深入 到武术课程教学中, 不仅对武术教师自身教学水平的 要求更加严格, 扮演的角色有了巨大的转变, 同时对 学生的武术学习也产生了积极地影响, 促进了学校体 育教学改革的发展, 其应用价值和意义主要通过以下 方面体现。

\section{1. 现代信息技术在武术教学中对教师的影 响}

\subsection{1 有助于促进武术教学方法的多样化}

随着现代信息技术不断的发展和我国教学改革 不断的深入, 现代信息技术进入武术教学课堂, 使得 教师在武术教学的方法上产生了巨大的改变, 教学的 方法不再是单一的 “注入式” 与 “填鸭式”。武术教 学不再是仅仅以讲解示范来完成, 而利用现代信息技 术辅助武术教学, 从视觉, 听觉等多方面诱导学生学 习, 更容易教师与学生之间的互动。在教学过程中教 师可以利用现代信息技术积极探索和创新, 教学手段、 方法不断丰富。

\subsection{2 有助于提高教师在现代信息技术环境下 的教学能力}

教师作为教育教学活动的组织者和主导者, 在 “互联网+” 的时代, 必然也是教育信息化进程的重 要推动者。教师不仅要改变教育理念, 掌握现代教育 信息技术, 还要不断提升自我的现代信息加工处理能 力。 ${ }^{[1]}$ 具体到武术教学, 武术教师不但要掌握专业的 武术技能, 还要不断学习现代信息技术的基本知识, 通过学习和培训, 掌握、提高使用多媒体等现代信息 技术进行武术教学的能力。只有教师的能力提升了, 信息化课堂教学的效果才能得到提高。

\subsection{3 有助于武术课堂中教师的角色调整}

信息化教学为教师提供了丰富的教学资源, 使教 师教学手段更加灵活, 在教学过程中, 教师是主要因 素之一, 在教学过程中应该如何调整自己的角色, 以 适应现代信息化课堂的教学的要求是目前最首要的
任务。信息化教学是必然的发展趋势, 教师必须重新 定位自己在教学中扮演的角色。

（1）教师是新型武术教学的环境创建与管理者

情境认知理论认为, 教学设计要以学生为主体, 教学的内容、教学活动的安排要和实践活动相连通, 把学习者获得知识的途径、方法手段、组织和身份建 构相结合。因此, 教师要根据教学实际情况, 在教学 过程中为学生创造适宜的学习环境并且进行管理, 保 证教学的正常进行。教师可以通过制作多媒体课件, 播放有关武术的视频, 让学生观看各种大型武术比赛 和各种武术表演, 在有条件的可以组织看直播等形式, 让学生走进武术的情境中。此外, 教师还可以通过网 络信息技术搜集相关武术素材, 制作微课微视频等, 引导学生观看学习并进行问题的互动解答, 拓展学生 的武术学习渠道。

（2）教师是学生学习武术过程中的导师

在现代信息技术构建的武术教学环境中，教师作 为知识的传递者的角色已经趋于过时, 强化了教师导 师的地位。现代信息技术具有双面性，一方面传递了 人类优秀的文化, 另一方面也夹杂着许多对学生学习 武术产生副作用的不利因素, 因此, 学生在学习的过 程中需要教师的指导。例如, 学生因为受到一些武侠 类书籍与影视中过于夸张的武术动作影响, 与现实中 的武术动作相比相差甚远，因此对武术产生质疑与偏 见，这时候就需要教师用现代信息技术来揭示艺术夸 张和现实武术表现得差异与不同，使他们以正确的眼 光看待武术。

（3）教师是学生学习武术过程中的伙伴

因为当今信息技术迅速的崛起，新的知识、新的 理论层出不穷, 大多教师与学生都处于同一起跑线, 教师在利用信息技术教学的同时也要努力学习, 以适 应信息化课堂教学模式, 掌握信息教学手段。在信息 化教学当中, 教师的理解能力强与经验丰富, 而学生 的记忆能力和适应能力比较强, 在教学过程中教师与 学生各持所长形成互补, 相互学习, 这时候教师的角 色就是一个学习者。教学过程教师不再是学生的权威 者、主宰者, 教师以平等的身份进行教学, 每个武术 教师自己的动作不一定就完全是正确的, 总有一些动 作不到位或者是动作衔接方面或多或少都有问题, 然 而现在利用现代信息技术教学, 在教学生的同时也能 发现自身的问题, 从而进行自我完善动作，提高自己 的教学水平, 作为学生的学习伙伴一起进行学习探讨, 共同解决问题。

\section{2. 现代信息技术在武术教学中对学生的影 响}

\section{2. 1. 帮助学生提高对武术的认知}

中小学生对于武术的了解主要源于武侠影视剧、 武侠小说、武术英雄人物事迹以及日常中的习武者的 
影响等方面, 了解武术的途径较窄, 知识面薄弱, 缺 少对武术理论知识和技能的较为全面的认知。在现代 信息技术与武术教学课堂相融合的背景下, 学生通过 信息技术手段来了解和学习武术知识理论和技能, 这 对于提高学生的武术认知水平和学习兴趣具有很大 帮助。

\subsection{2. 便于学生建立正确的武术动作概念}

武术技术动作结构复杂、难度动作多、动作线路 变换多, 在武术教学中, 教师除了讲解和示范单个动 作技术外, 还要进行完整的示范, 对于年纪大或身体 原因无法完成较高难度武术动作的教师而言, 利用现 代信息技术教学就非常有用, 教师可以通过多媒体技 术, 利用视频慢放、图片的移动、旋转来演示武术动 作的运动轨迹、完成过程及身体在空间的姿势, 把武 术动作要点、细节展现在学生面前, 使学生建立了正 确的武术动作概念。同时, 在武术演练时通过配音乐 方式来烘托气氛, 录像讲评等来提高武术教学水平。

\section{3. 促进武术文化的传承和弘扬}

武术是优秀的中华民族传统体育项目, 是传统文 化的重要载体, 需要我们代代传承。现代信息技术具 有传递信息与存储信息的功能, 它能够将中华武术文 化和精神推广向全世界, 从而促进中华民族传统体育 精神的传承和弘扬。学校武术教学是弘扬和传承武术 文化的重要方式之一, 在教学中利用现代信息技术使 学生能够广泛的了解武术文化, 迅速的获得武术知识 技能。教师可通过信息技术加强对学生的武术学习环 节的指导, 利用现代信息技术丰富教学内容以及形式, 并通过与实践活动相结合来进行综合教学的方式, 激 发学生对武术文化学习的兴趣, 在学生心中播撒中华 传统文化的种子。

\section{4. 促进武术信息化课堂的开发与应用}

随着新课改的深入, 现代信息技术迅速的发展, 现代教学课堂引入现代信息技术为教师提供了便捷 有效的教学手段和丰富的教学资源, 使教学方法多元 化, 教学资源的开发与运用的规范化。我国传统的武 术教学方法单一, 教学手段单调, 教学资源利用率 低……现代信息化课堂开发与管理, 可以有效改变这 种情况, 大幅度提升教学资源的利用水平, 构建新的 教学模式。信息技术在武术教学中的应用, 加速了武 术教学课堂信息化的进程。

\section{3. 在现代信息技术背景下中小学武术教学方 法改革}

\section{1. 利用现代信息技术激发学生武术学习的 兴趣}

传统的武术教学方法讲解、示范为主, 优点就是
直观、师生亲力亲为, 相互交流直接。不足之处在于, 复杂的、有难度的一些动作无法对学生进行清晰的展 示，同时，教师个人武术知识的局限性也会影响到武 术教学。但是，把传统武术教学同现代信息技术相结 合, 就能够解决这样的问题。我们可以利用现代信息 技术手段, 收集武术文化知识推介给学生; 对难以掌 握的、复杂的动作技术，教师可以制作教学视频、采 用多方位视角给学生进行展示, 使学生了解动作结构; 还可通过信息互动解答学生的问题; 通过播放武术竞 赛及武术影视使学生更广泛的了解武术……通过这 些形式, 使学生能够全方位的进入到武术学习场景中, 从而激发出学生的武术学习兴趣。

\section{2. 在武术理论教学中广泛的应用现代信息 技术}

精彩的体育理论教学对教师而言是一项具有挑 战性的事情。枯燥干澀的体育理论往往影响到学生学 习的积极性。在现代信息技术条件下，教师可通过运 用信息技术来提高教学质量与效率, 从而达成教学目 标。 ${ }^{[3]}$ 其一, 教师根据教学内容制作出教学课件, 结 合以图片、影像、图表等方式, 呈现出教学内容并进 行讲授; 其二, 通过互联网系统收集武术相关的文献 资料对教学内容进行补充, 拓展学生的知识空间; 其 三, 通过组织欣赏线上武术竞赛和武术演练等视频, 讲解武术竞赛规则及武术演练知识, 提高学生对武术 的认知; 其四, 通过远程辅助武术教学, 师生互动等 形式, 使学生能够获得学习上的指导与帮助, 提高学 生的武术理论知识水平。

\section{3. 在武术技术教学中合理的应用现代信息 技术}

由于武术运动本身具有的特性, 教学中需要教师 以示范、领做为主要教学方法, 但是对于目前中小学 课堂来看, 课堂人数多, 这对于教师的示范又提出了 难题, 示范过程中很多学生因为前边学生阻挡或者距 离太远而看不清楚动作, 就需要教师不断进行重复的 讲解示范, 有的教师在进行示范时, 因为自身因素限 制, 一些动作可能无法完成, 这些都会影响到教学效 果。因此, 要提高教学效果, 重要一点在于教师要合 理的解析教材的难点、重点, 利用多媒体播放标准动 作示范, 动作快慢可以由教师控制, 还可以多个角度 进行观看, 教师一边播放视频一边完成技术动作要领 讲解, 这样教学中的困难、复杂动作教学难点问题就 得到了解决。 ${ }^{[5]}$ 在教学过程中纠错也是一个难点问题, 在学习中可利用拍摄工具录制学生在练习过程中的 图片与视频, 然后在给学生们观看自己练习时候的图 片与视频, 与标准的视频动作进行比较, 让学生自己 找自己动作的问题, 教师再讲解帮助学生如何纠正动 作, 使学生对武术动作印象更加深刻。当然, 仅仅是 课堂上的时间学生是不可能把武术动作完全掌握的, 教师可以利用通讯工具或者是存储工具将视频影像 
等学习资料发送给学生, 使学生便于课后自主学习完 成武术动作，从而提升教学的效率。通过信息技术， 教师还可以对学生自主学习动作之后录制的视频进 行评价互动, 进一步对学生动作进行指导纠正, 使教 学达到预期结果, 保证教学的质量。

\section{4. 利用现代信息技术使武术教学资源共享}

传统的武术教学在多方面受到人力、技术等方面 的制约, 导致我国中小学武术教学的知识内容、信息 都受到了限制, 因此, 中小学武术教学难以享受广泛 的武术教学资源。但是当今的中国, 信息网络技术发 达, 人们充分的利用网络技术, 能够随时获得来自各 地的、广泛的、优质的武术教学资源。利用电脑、手 机等互联网工具都可以在最短的时间内, 获得各地的 先进的教学理论与方法方面的科研成果 ${ }^{[3]}$ 。现代信息 技术在武术教学中的应用, 使广大武术教师和学生的 视野得到了拓展，也使武术教学资源的共享更加便捷。

\section{4. 结论}

现代信息技术在武术教学中的应用, 为武术教学 提供了丰富的教学资源, 拓展了武术教学手段与方法, 促进了武术教学方法、形式与资源的共享, 推动了武 术教学方法不断改革与创新, 对武术教师和学生都产 生了巨大的影响。

现代信息技术在武术教学中的应用, 是对传统武 术教学方法的拓展与补充, 开拓了武术的发展空间, 同时也给广大武术教师的知识结构、教学能力和水平 提出了更高的要求。

\section{项目基金}

本文为陕西省教育科学 “十三五” 规划 2018 年 度课题（课题编号：SGH18H363）。

\section{REFERENCES}

[1] Yao Meilin. (2003)From Cognition to Situation: the Change of Learning Paradigm. J. Journal of Beijing Normal University, 2: 3-4.

[2] Zhang Jing. (2005) Wushu Teaching, Cultivation and Promotion of National Spirit in Primary and Secondary Schools. J. Fighting. Wushu science, 12: 73-74.

[3] Zhong Suiping. (2006) Application of Modern Educational Technology to Promote teaching Reform .J. China Medical Educational Technology, 4:52-54.

[4] Liu Zhongdong. (2009) Research on mathematical Understanding based on situational cognition and learning Theory .J. Journal of Jinggangshan University, 5: 51-52.
[5] Sun Jin. (2013) Modern Information Technology promotes the Leapfrog Development of School PHYSICAL Education .J. Middle School Teaching Reference, 11: 103-105. 